\title{
A Note on the Construction of Constant Order Predictor Corrector Algorithm for the Solution of $y^{\prime}=f(x, y)$
}

\author{
James A.A ${ }^{1 *}$ and Adesanya A.O. ${ }^{2}$ \\ ${ }^{1}$ Mathematics Division, American University of Nigeria. Yola, Adamawa State, Nigeria. \\ ${ }^{2}$ Mathematics Department, Modibbo Adama University of Technology Yola, Adamawa State,
}

Nigeria.

\begin{tabular}{l}
$\begin{array}{l}\text { Original Research } \\
\text { Article }\end{array}$ \\
\hline
\end{tabular}

Received: 06 August 2013

Accepted: 28 September 2013

Published: 16 January 2014

\section{Abstract}

This paper examines the development of one step, five hybrid point method for the solution of first order initial value problems. We adopted the method of collocation and interpolation of power series approximate solution to generate a continuous linear multistep method. The continuous linear multistep method was evaluated at selected grid points to give the discrete linear multistep method. The method was implemented using a constant order predictor of order seven over an overlapping interval. The basic properties of the derived corrector was investigated and found to be zero stable, consistent and convergent. The region of absolute stability was also investigated. The method was tested on some numerical experiments and found to compare favorably with the existing methods.

Keywords: constant order, hybrid points, collocation, interpolation, approximate solution, grid points, zero stable, consistent, convergent.

AMS Subject Classification (2010): 65L05, 65L06, 65D30

\section{Introduction}

It is remarkable to note that many physical phenomena in sciences, engineering, and medicine, to mention few, are modeled by equations involving derivatives, which are generally referred to as differential equations. A differential equation in which the unknown parameter is a function of one independent variable is called an ordinary differential equations, while that involving two or more independent variables is called a partial differential equation.

The general form of the initial value problems of ordinary differential equations is in the form;

$$
y^{(n)}=f\left(x, y, y^{(1)}, \ldots y^{(n-1)}\right), y(a)=\varphi_{0}, \ldots, y^{(n-1)}(a)=\varphi_{n-1} .
$$

\footnotetext{
*E-mail: adewale.james@aun.edu.ng
} 
In most cases, modeled problems do not have analytical solutions; hence numerical methods are often the only option to solve such problems.

Many physical problems are modeled into first order ordinary differential equations, the few that are modeled into higher order ordinary differential equations, are solved by reducing them to a system of first order ordinary differential equations. Hence, the study of first order ordinary differential equation is important. This paper considers a numerical method of solving first order initial value problems of ordinary differential equations of the form;

$$
y^{\prime}=f(x, y), y(a)=y_{0}, a \leq x \leq b<\infty
$$

where $f(x, y)$ is a given real valued function in the strip $S=[a, b] \subset[-\infty, \infty]$, which is continuous within the region. We assumed that $f(x, y)$ satisfies Lipchitz conditions that guaranteed the existence and uniqueness of the solution to equation 1.2.

Scholars have developed linear multistep method for the solution of 1.1. They developed methods varying from the discrete linear multistep method to the continuous linear multistep method. According to [1], the continuous linear multistep method has greater advantages over the discrete method, in that it gives better error estimation, provides a simplified form of coefficient for further analytical work at different points, and guarantees easy appropriation of solutions at all interior points within the interval of integration. Among the authors that proposed the continuous linear multistep method are; [2],[3],[4], to mention a few. They individually proposed methods which are implemented in predictor corrector mode, and adopted Taylor series expansion to supply the starting value.

Generally, the major setback of the predictor-corrector method is the high cost of implementation, as subroutines are very complicated to write because of the special techniques required to supply starting values. Therefore we seek to address this setback by proposing a method that shares the properties of both the block method and the predictor corrector method. It should be recalled that [5] first proposed block method as a predictor to a predictor corrector algorithm. [6], [7],[8] adopted the Milne's approach and concluded that though the method is more expensive to implement but it gives better results than the block method; hence the method follows the Milne's approach.

\section{Methods and Materials}

\subsection{Derivation of the Corrector}

We consider a power series approximate solution in the form

$$
y(x)=\sum_{j=0}^{s+r-1} a_{j} x^{j},
$$

where $r$ and $s$ are the number of interpolation and collocation points respectively. Substituting the first derivative of 2.1 into 1.2 gives

$$
f(x, y)=\sum_{j=1}^{s+r-1} j a_{j} x^{j-1} .
$$

Interpolating 2.1 at $x_{n}, x_{n+\frac{1}{6}}, x_{n+\frac{1}{3}}$ and collocating 2.2 at $x_{n+s}, s=0\left(\frac{1}{6}\right) 1$ gives a system of non linear equation in the form

$$
A X=U
$$


where

$$
\begin{aligned}
& A=\left[\begin{array}{lllllllll}
a_{0} & a_{1} & a_{2} & a_{3} & a_{4} & a_{5} & a_{6} & a_{7} & a_{8}
\end{array}\right]^{T}, \\
& U=\left[\begin{array}{lllllll}
y_{n} & y_{n+\frac{1}{6}} & y_{n+\frac{1}{3}} & f_{n} f_{n+\frac{1}{3}} f_{n+\frac{1}{2}} f_{n+\frac{2}{3}} f_{n+\frac{5}{6}} f_{n+1}
\end{array}\right]^{T}, \\
& X=\left[\begin{array}{cccccccccc}
1 & x_{n} & x_{n}^{2} & x_{n}^{3} & x_{n}^{4} & x_{n}^{5} & x_{n}^{6} & x_{n}^{7} & x_{n}^{8} & x_{n}^{9} \\
1 & x_{n+\frac{1}{6}} & x_{n+\frac{1}{6}}^{2} & x_{n+\frac{1}{6}}^{3} & x_{n+\frac{1}{6}}^{4} & x_{n+\frac{1}{6}}^{5} & x_{n+\frac{1}{6}}^{6} & x_{n+\frac{1}{6}}^{7} & x_{n+\frac{1}{6}}^{8} & x_{n+\frac{1}{6}}^{9} \\
1 & x_{n+\frac{1}{3}} & x_{n+\frac{1}{3}}^{2} & x_{n+\frac{1}{3}}^{3} & x_{n+\frac{1}{3}}^{4} & x_{n+\frac{1}{3}}^{5} & x_{n+\frac{1}{3}}^{6} & x_{n+\frac{1}{3}}^{7} & x_{n+\frac{1}{3}}^{8} & x_{n+\frac{1}{3}}^{9} \\
0 & 1 & 2 x_{n} & 3 x_{n}^{2} & 4 x_{n}^{3} & 5 x_{n}^{4} & 6 x_{n}^{5^{3}} & 7 x_{n}^{6} & 8 x_{n}^{7} & 9 x_{n}^{8} \\
0 & 1 & 2 x_{n+\frac{1}{6}} & 3 x_{n+\frac{1}{6}}^{2} & 4 x_{n+\frac{1}{6}}^{3} & 5 x_{n+\frac{1}{6}}^{4} & 6 x_{n+\frac{1}{6}}^{5} & 7 x_{n+\frac{1}{6}}^{6} & 8 x_{n+\frac{1}{6}}^{7} & 9 x_{n+\frac{1}{6}}^{8} \\
0 & 1 & 2 x_{n+\frac{1}{3}} & 3 x_{n+\frac{1}{3}}^{2} & 4 x_{n+\frac{1}{3}}^{3} & 5 x_{n+\frac{1}{3}}^{4} & 6 x_{n+\frac{1}{3}}^{5} & 7 x_{n+\frac{1}{3}}^{6} & 8 x_{n+\frac{1}{3}}^{7} & 9 x_{n+\frac{1}{3}}^{8} \\
0 & 1 & 2 x_{n+\frac{1}{2}} & 3 x_{n+\frac{1}{2}}^{2} & 4 x_{n+\frac{1}{2}}^{3} & 5 x_{n+\frac{1}{2}}^{4} & 6 x_{n+\frac{1}{2}}^{5} & 7 x_{n+\frac{1}{2}}^{6} & 8 x_{n+\frac{1}{2}}^{7} & 9 x_{n+\frac{1}{2}}^{8} \\
0 & 1 & 2 x_{n+\frac{2}{3}} & 3 x_{n+\frac{2}{3}}^{2} & 4 x_{n+\frac{2}{3}}^{3} & 5 x_{n+\frac{2}{3}}^{4} & 6 x_{n+\frac{2}{3}}^{5} & 7 x_{n+\frac{2}{3}}^{6} & 8 x_{n+\frac{2}{3}}^{7} & 9 x_{n+\frac{2}{3}}^{8} \\
0 & 1 & 2 x_{n+\frac{5}{6}} & 3 x_{n+\frac{5}{6}}^{2} & 4 x_{n+\frac{5}{6}}^{3} & 5 x_{n+\frac{5}{6}}^{4} & 6 x_{n+\frac{5}{6}}^{5} & 7 x_{n+\frac{5}{6}}^{6} & 8 x_{n+\frac{5}{6}}^{7} & 9 x_{n+\frac{5}{6}}^{8} \\
0 & 1 & 2 x_{n+1} & 3 x_{n+1}^{2} & 4 x_{n+1}^{3} & 5 x_{n+1}^{4} & 6 x_{n+1}^{5} & 7 x_{n+1}^{6} & 8 x_{n+1}^{7} & 9 x_{n+1}^{8}
\end{array}\right]
\end{aligned}
$$

Solving 2.3 for $a_{j}^{\prime} s$ using Gaussian elimination method gives a continuous hybrid linear multistep method in the form

$$
y(t)=\alpha_{\mu}(t) y_{n+\mu}+h\left(\sum_{j=0}^{1} \beta(t) f_{n+j}+\beta_{k}(t) f_{n+k}\right),
$$

where $\mu=0, \frac{1}{6}, \frac{1}{3}, k=\frac{1}{6}\left(\frac{1}{6}\right) \frac{5}{6}, f_{n+k}=f\left(\left(x_{n}+k h\right), y\left(x_{n}+k h\right)\right), t=\frac{x-x_{n}}{h}$

$$
\left.\begin{array}{l}
\alpha_{0}=\frac{1}{58879}\left(\begin{array}{c}
1105397280 t^{9}-4654320912 t^{8}+8116009488 t^{7}-7598367000 t^{6}+ \\
4136522418 t^{5}-1320624081 t^{4}+233757738 t^{3}-18630810 t^{2}+58879
\end{array}\right), \\
\alpha_{\frac{1}{6}}=\frac{1}{58879}\left(\begin{array}{c}
3224862720 t^{9}-12733168896 t^{8}+20296479744 t^{7}-16688851200 t^{6}+ \\
7465893120 t^{5}-1734421248 t^{4}+170636544 t^{3}-2177280 t^{2}
\end{array}\right), \\
\alpha_{\frac{1}{3}}=\frac{1}{58879}\left(\begin{array}{c}
-4330260000 t^{9}+17387489808 t^{8}-28412489232 t^{7}+24287218200 t^{6} \\
-11602415538 t^{5}+3055045329 t^{4}-404394282 t^{3}+20808090 t^{2}
\end{array}\right), \\
\beta_{0}=\frac{1}{24729180}\left(\begin{array}{c}
20094564240 t^{9}-85453263360 t^{8}+151143614712 t^{7} \\
-144534487104 t^{6}+81350668413 t^{5}-27486167940 t^{4}+ \\
5429454157 t^{3}-572556798 t^{2}+24729180 t
\end{array}\right), \\
\beta_{\frac{1}{6}}=\frac{1}{2060765}\left(\begin{array}{c}
17436863520 t^{9}-71370079776 t^{8}+119715654888 t^{7}-106136094960 t^{6} \\
+53407542888 t^{5}-15160023648 t^{4}+2232970848 t^{3}-130340160 t^{2}
\end{array}\right), \\
\beta_{\frac{1}{3}}=\frac{9}{1648612}\left(\begin{array}{c}
11737401552 t^{9}-46356200208 t^{8}+74148407640 t^{7}-61663976136 t^{6} \\
+28457215353 t^{5}-7197296085 t^{4}+917530839 t^{3}-45746955 t^{2} \\
3868440 t^{2}-82463032 t^{3}+697706352 t^{4}-3014282376 t^{5}+
\end{array}\right), \\
\beta_{\frac{2}{3}}=\frac{1}{1236459}\left(\begin{array}{c}
7157930472 t^{6}-9342961200 t^{7}+6255519840 t^{8}-1674530496 t^{9} \\
1648612
\end{array}\right), \\
\beta_{\frac{5}{6}}=-\frac{1}{2060765}\left(\begin{array}{c}
14921552 t^{9}-2534167296 t^{8}+3573835992 t^{7}-2585556288 t^{6}+ \\
1036115577 t^{5}-230472900 t^{4}+26422173 t^{3}-1211310 t^{2} \\
199104480 t^{9}-668113920 t^{8}+895423896 t^{7}-621418392 t^{6}+ \\
241167024 t^{5}-52370640 t^{4}+5898456 t^{3}-266904 t^{2}
\end{array}\right), \\
\beta_{1}=\frac{1}{24729180}\left(\begin{array}{c}
254100240 t^{9}-804781008 t^{8}+1034093304 t^{7}-695981160 t^{6} \\
+264161709 t^{5}-56446929 t^{4}+6283999 t^{3}-281955 t^{2}
\end{array}\right),
\end{array}\right),
$$


Evaluating 2.4 at $t=1$ gives

$$
\begin{aligned}
& y_{n+1}-\frac{197000}{58879} y_{n}-\frac{746496}{58879} y_{n+\frac{1}{6}}+\frac{1002375}{58879} y_{n+\frac{1}{3}} \\
& =h\left(\begin{array}{c}
-\frac{172225}{1236459} f_{n}-\frac{701280}{412153} f_{n+\frac{1}{6}}-\frac{666000}{412153} f_{n+\frac{1}{3}}+\frac{788000}{1236459} f_{n+\frac{1}{2}} \\
-\frac{28125}{412153} f_{n+\frac{2}{3}}+\frac{115200}{412153} f_{n+\frac{5}{6}}+\frac{57410}{1236459} f_{n+1}
\end{array}\right),
\end{aligned}
$$

equation 2.5 is our corrector.

\subsection{Derivation of the Constant Order Predictor}

[8] had developed a block method which we adopted as our constant order predictor. They considered collocating 2.2 at $x_{n+s, s}=0\left(\frac{1}{6}\right) 1$ and interpolating 2.1 at $x_{n}$ to obtained a discrete block method given as

$$
\mathbf{Y}_{m}=\mathbf{e} y_{n}+h \mathbf{d} f\left(y_{n}\right)+h \mathbf{b f}\left(\mathbf{Y}_{m}\right),
$$

where,

$$
\begin{aligned}
\mathbf{d} & =\left[\frac{19087}{362880} \frac{1139}{22680} \frac{137}{2688} \frac{143}{2835} \frac{3715}{72576} \frac{41}{840}\right]^{T} \mathbf{Y}_{m}=\left[y_{n+\frac{1}{6}} y_{n+\frac{1}{3}} y_{n+\frac{1}{2}} y_{n+\frac{2}{3}} y_{n+\frac{2}{3}} y_{n+\frac{5}{6}} y_{n+1}\right]^{T}, \\
\mathbf{b} & =\left[\begin{array}{ccccccc}
\frac{2713}{13120} & \frac{-15487}{120960} & \frac{293}{2835} & \frac{-6737}{120960} & \frac{263}{15120} & \frac{-863}{36288} \\
\frac{47}{189} & \frac{11}{7560} & \frac{166}{2835} & \frac{-269}{7560} & \frac{11}{945} & \frac{-37}{22680} \\
\frac{27}{112} & \frac{387}{4480} & \frac{17}{105} & \frac{-243}{4480} & \frac{9}{560} & \frac{-29}{13440} \\
\frac{232}{945} & \frac{64}{945} & \frac{752}{2835} & \frac{29}{945} & \frac{8}{945} & \frac{-4}{2835} \\
\frac{725}{3024} & \frac{2125}{24192} & \frac{125}{567} & \frac{2875}{24192} & \frac{235}{3024} & \frac{-275}{72516} \\
\frac{9}{35} & \frac{9}{280} & \frac{34}{105} & \frac{9}{280} & \frac{9}{35} & \frac{41}{840}
\end{array}\right] \quad \mathbf{e}=\left[\begin{array}{cccccc}
0 & 0 & 0 & 0 & 0 & 1 \\
0 & 0 & 0 & 0 & 0 & 1 \\
0 & 0 & 0 & 0 & 0 & 1 \\
0 & 0 & 0 & 0 & 0 & 1 \\
0 & 0 & 0 & 0 & 0 & 1 \\
0 & 0 & 0 & 0 & 0 & 1
\end{array}\right],
\end{aligned}
$$

kindly refer to [8] for the analysis of the basic properties of this method.

\subsection{Implementation of the Method}

In order to implement the method, we propose a prediction equation of the form

$$
Y_{m}^{(0)}=(j h) y_{n}+h \sum_{\lambda=0}^{2} \frac{\partial \lambda}{\partial x^{\lambda}} f(x, y)_{\left(x_{0}, y_{0}\right)},
$$

substituting 2.7 into the general block formula (8) gives

$$
Y_{m}=e y_{n}+h\left[d f\left(y_{n}\right)+b F\left(Y_{m}^{(0)}\right)\right],
$$

Writing 2.5 in a linearized form gives

$$
Y_{N+1}=Y_{N+\mu}+h\left[b F\left(Y_{N+\mu}\right)\right],
$$

where $\mu$ is the grid points, $Y_{N+\mu}$ are the interpolation points and $F\left(Y_{N+\mu}\right)$ are the collocation points, hence substituting 2.8 into 2.9 gives

$$
Y_{N+1}=Y_{N+\mu}+h\left[b F\left(Y_{m}\right)\right],
$$

where $Y_{m}=Y_{N+\mu} .2 .10$ is our new method 


\section{Analysis of the basic Properties of the Corrector}

\subsection{Order and Error Constant of the Corrector}

Let the linear operator $\ell\{y(x) ; h\}$ associated with the hybrid linear multistep method be defined as

$$
\left.\ell\{y(x) ; h\}=y(x)-\alpha_{\mu}(x) y_{n+\mu}+h \sum_{j=0}^{1} \beta(x) f_{n+j}+\beta_{k}(x) f_{n+k}\right) .
$$

Expanding $\ell\{y(x) ; h\}$ in Taylor series and comparing the coefficient of $h$ gives

$$
\ell\{y(x): h\}=\left(\begin{array}{c}
C_{0} y(x)+C_{1} h y^{\prime}(x)+C_{2} h^{2} y^{\prime \prime}(x)+\ldots+C_{p} h^{p} y^{p}(x) \\
+C_{p+1} h^{p+1} y^{p+1}(x)+C_{p+2} h^{p+2} y^{p+2}(x)+\ldots
\end{array}\right)
$$

Definition 1 The linear operator $\ell$ and the associated continuous linear multistep method 2.5 is said to be of order $p$ if $C_{O}=C_{1}=C_{2}=\ldots=C_{P}=0$ and $C_{p+1} \neq 0 . C_{p+1}$ is called the error constant and implies that the local truncation error is given by

$$
t_{n+k}=C_{p+1} h^{p+1} y^{p+1}(x)+O\left(h^{p+2}\right) t_{n+k},
$$

For our corrector, $C_{0}=C_{1}=\ldots=C_{9}=0, C_{10} \neq 0$ hence the order of the method is 9 with error constant $c_{p+1}=-4.8753 \times 10^{-11}$

\subsection{Zero Stability of the Corrector}

A Continuous hybrid linear multistep method is said to be zero stable, if the zeros of the first characteristic polynomial $\sigma(r)$ satisfies $|r|=1$ is simple.

The first characteristics polynomial of 2.5 is given

$$
\rho(z)=z-\frac{197000}{58879}-\frac{746496}{58879} z^{\frac{1}{6}}+\frac{1002375}{58879} z^{\frac{1}{3}}
$$

equating 3.1 to zero and solving for $z$ gives the roots of the first characteristic polynomial as 0 and 1 , hence our corrector is zero stable

\subsection{Consistency of the Corrector}

A method is said to be consistent if

- it has order $p \geq 1$

- if $\varrho(1)=\varrho^{\prime}(1)=\ldots \varrho^{(n-1)}(1)=0$, where $\varrho(z)$ is the first characteristic polynomial, $n$ is the order of the differential equation.

- if $\varrho^{(n)}(1)=n ! \sigma(1)$ where $\sigma(z)$ is the second characteristic polynomial.

The second characteristic polynomials of 2.5 is given as

$$
\begin{gathered}
\sigma(r)=-\frac{172225}{1236459}-\frac{701280}{412153} z^{\frac{1}{6}}-\frac{666000}{412153} z^{\frac{1}{3}}+\frac{788000}{1236459} z^{\frac{1}{2}}-\frac{28125}{412153} z^{\frac{2}{3}} \\
+\frac{115200}{412153} z^{\frac{5}{6}}+\frac{57410}{1236459} z,
\end{gathered}
$$

$\rho(1)=0, \rho^{\prime}(1)=\sigma(1)$ hence our method is consistent. 


\subsection{Convergence}

The necessary and sufficient condition for a linear multistep method to be convergent is that it must be consistent and zero stable. Hence our corrector is convergent.

\subsection{Region of Absolute Stability of our Corrector}

Definition: A method is said to be absolutely stable if for a given value of $h$, all the roots $z_{s}$ of the characteristics polynomial $\Pi(z, \bar{h})=\rho(z)+\bar{h} \sigma(z)=0$, satisfying $\left|z_{s}\right|<s, s=1,2 \ldots, n$ where $\bar{h}=$ $\lambda h, \lambda=\frac{d f}{d y}$, substituting the test equation $y^{\prime}=\lambda y$ into (7), solving for $\bar{h}=\lambda h$ and writing $r=e^{i \theta}$, gives the stability region as shown in Fig. (1)

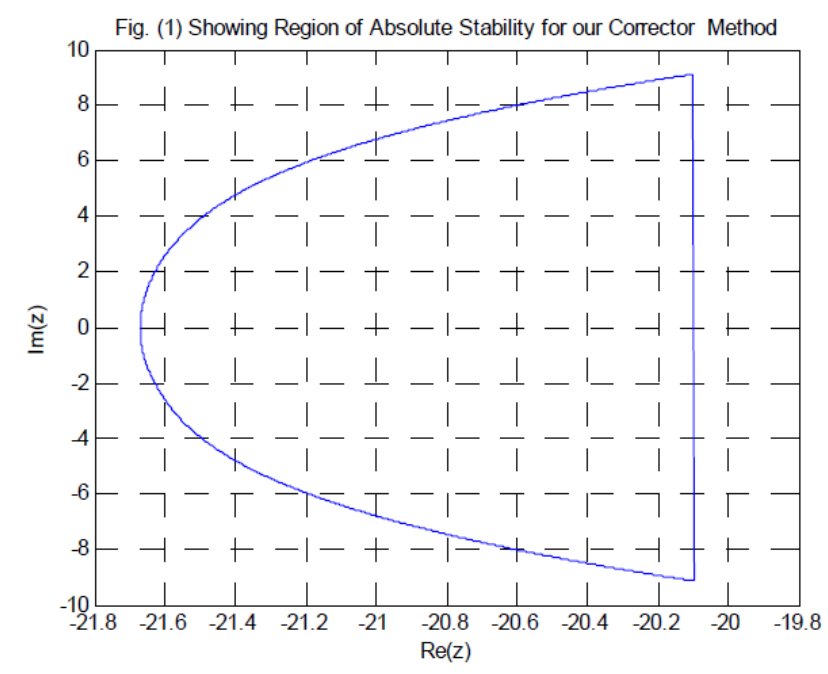

\section{Numerical Experiment}

\section{Problem 1}

$y^{\prime}=x-y, y(0)=0,0 \leq x \leq 1, h=0.1$

Exact solution: $y(x)=x+e^{-x}-1$

$\mathrm{ERB} \rightarrow$ Error in Block-predictor

ER1 $\rightarrow$ Error considering two interpolation

ERN $\rightarrow$ Error in New Method

ERA $\rightarrow$ Error in [9] 
Table 1 showing results generated from problem 1

\begin{tabular}{ccccc}
\hline$x$ & $E R B ;$ & $E R 1$ & $E R N$ & $E R A$ \\
\hline 0.1 & $1.9595(-11)$ & $2.26688(-13)$ & $9.1770(-14)$ & 0.000 \\
0.2 & $3.54623(-11)$ & $2.04759(-13)$ & $8.2666(-14)$ & 0.000 \\
0.3 & $4.81315(-11)$ & $1.84879(-13)$ & $7.4419(-14)$ & $6.0(-10)$ \\
0.4 & $5.80680(-11)$ & $1.67213(-13)$ & $6.7335(-14)$ & $2.0(-10)$ \\
0.5 & $6.56779(-11)$ & $1.50879(-13)$ & $6.0326(-14)$ & $7.0(-10)$ \\
0.6 & $7.13132(-11)$ & $1.36446(-13)$ & $5.4511(-14)$ & $1.0(-10)$ \\
0.7 & $7.52814(-11)$ & $1.23123(-13)$ & $4.9016(-14)$ & $8.0(-10)$ \\
0.8 & $7.78485(-11)$ & $1.11272(-13)$ & $4.4492(-14)$ & $2.0(-10)$ \\
0.9 & $7.92403(-11)$ & $1.00697(-13)$ & $3.9857(-14)$ & $9.0(-10)$ \\
1.0 & $7.96712(-11)$ & $9.11493(-13)$ & $3.6415(-14)$ & $4.0(-10)$ \\
\hline
\end{tabular}

\section{Problem 11:}

$y^{\prime}=-y, y(0)=1,0 \leq x \leq 1, h=0.1$.

Exact solution $y(x)=e^{-x}$

ERUY $\rightarrow$ Error in [10]

Table 2: Showing results generated from Problem 11

\begin{tabular}{ccccc}
\hline$x$ & $E R B$ & $E R 1$ & $E R N$ & $E R U Y$ \\
\hline 0.1 & $1.9596(-11)$ & $2.2648(-13)$ & $9.3702(-14)$ & $2.5292(-06)$ \\
0.2 & $3.5462(-11)$ & $2.04614(-13)$ & $8.2489(-14)$ & $2.0937(-06)$ \\
0.3 & $4.8131(-11)$ & $1.85074(-13)$ & $7.4606(-14)$ & $2.0079(-06)$ \\
0.4 & $5.8068(-11)$ & $1.67199(-13)$ & $6.7390(-14)$ & $1.6198(-06)$ \\
0.5 & $6.5677(-11)$ & $1.51212(-13)$ & $6.0729(-14)$ & $3.1608(-06)$ \\
0.6 & $7.1313(-11)$ & $1.36668(-13)$ & $5.3179(-14)$ & $2.7294(-06)$ \\
0.7 & $7.5281(-11)$ & $1.23179(-13)$ & $4.8072(-14)$ & $2.5457(-06)$ \\
0.8 & $7.7848(-11)$ & $1.11188(-13)$ & $4.3687(-14)$ & $2.1713(-06)$ \\
0.9 & $7.9245(-11)$ & $1.00530(-13)$ & $4.0578(-14)$ & $3.1008(-06)$ \\
1.0 & $7.9671(-11)$ & $9.05386(-14)$ & $3.5527(-14)$ & $2.7182(-06)$ \\
\hline
\end{tabular}

Problem 111:

We Consider the growth model described by the differential equation of the form

$$
\frac{d N}{d t}=\alpha N, N(0)=1000, t \in[0,1]
$$

The above growth equation represents the rate of growth of bacteria in a colony. We shall assume that the model grows continuously without restriction. One may ask; how many bacteria are in a colony after some minutes if an individual produces an offspring at an average growth rate of 0.2 ? We also assume that $N(t)$ is the population size at time $t$.

The theoretical solution is given by

$N(t)=1000 e^{0.2 t}$, we note that the growth rate $\alpha=0.2$ in the growth equation.

$\mathrm{ERN} \rightarrow$ Error in New method

ERS $\rightarrow$ Error in [11] 
Table 3: Showing results generated from problem 111

\begin{tabular}{ccccc}
\hline$x$ & Exact result & Computed result & $E R N$ & $E R S$ \\
\hline 0.1 & 1020.2013400267558 & 1020.2013400267565 & $6.82121(-13)$ & $1.8303(-11)$ \\
0.2 & 1040.8107741923882 & 1040.8107741923861 & $2.04636(-12)$ & $1.2505(-11)$ \\
0.3 & 1061.8365465453596 & 1061.8365465453599 & $2.27373(-13)$ & $1.2278(-11)$ \\
0.4 & 1083.2870676749587 & 1083.2870676749576 & $1.13686(-12)$ & $3.1377(-11)$ \\
0.5 & 1105.1709180756477 & 1105.1709180756473 & $4.54747(-13)$ & $2.2168(-10)$ \\
0.6 & 1127.4968515793757 & 1127.4968515793755 & $2.27373(-13)$ & $2.0600(-10)$ \\
0.7 & 1150.2737988572273 & 1150.2737988572242 & $3.18323(-12)$ & $2.1714(-10)$ \\
0.8 & 1173.5108709918102 & 1173.5108709918097 & $4.54747(-13)$ & $2.2168(-10)$ \\
0.9 & 1197.2173631218104 & 1197.2173631218095 & $9.09494(-13)$ & $2.7444(-10)$ \\
1.0 & 1221.4027581601702 & 1221.4027581601722 & $2.04636(-12)$ & $4.8999(-10)$ \\
\hline
\end{tabular}

\section{Problem IV.}

The SIR model is an epidemiological model that computes the theoretical numbers of people infected with a contagious illness in a closed population over time. The name of this class of models derives from the fact that they involves coupled equations relating the number of susceptible people $\mathrm{S}(\mathrm{t})$, number of people infected $\mathrm{I}(\mathrm{t})$ and the number of people who have recovered $\mathrm{R}(\mathrm{t})$. This is a good and simple model for many infectious diseases including measles. The SIR model is described by the three coupled equations.

$$
\begin{aligned}
& \frac{d s}{d t}=\mu(1-S)-\beta I S \\
& \frac{d I}{d t}=-\mu I-\gamma I+\beta I S \\
& \frac{d R}{d t}=-\mu R+\gamma I
\end{aligned}
$$

Where $\mu, \gamma$ and $\beta$ are positive parameters. Define $y$ to be $y=S+I+R$ for

Adding equations the three coupled equations above, we obtain the following evolution equations

$$
y^{\prime}=\mu(1-y)
$$

Taking $\mu=0.5$ and attaching an initial condition $y(0)=0.5$ (for a particular closed population), we obtain,

$$
\begin{aligned}
& y^{\prime}(t)=0.5(1-y), y(0)=0.5 \\
& \text { Whose exact solution is, } \\
& y(t)=1-0.5 e^{-0.5 t}
\end{aligned}
$$

ERB-Error in Block method

ERN-Error in New method

ERS-Error in [11] 
Table 4: Showing results generated from problem IV

\begin{tabular}{ccccc}
\hline$x$ & Exact Results & $E R B$ & $E R N$ & $E R S$ \\
\hline 0.1 & 0.5243852877496430 & $7.704948 e-014$ & $3.33066(-16)$ & $5.574430 e-012$ \\
0.2 & 0.5475812909820202 & $1.465494 e-013$ & $6.66133(-16)$ & $3.946177 e-012$ \\
0.3 & 0.5696460117874711 & $2.090550 e-013$ & 0.0000000 & $8.183232 e-012$ \\
0.4 & 0.5906346234610092 & $2.652323 e-013$ & $3.33066(-16)$ & $3.436118 e-011$ \\
0.5 & 0.6105996084642975 & $3.151923 e-013$ & $1.11022(-15)$ & $1.929743 e-010$ \\
0.6 & 0.6295908896591411 & $3.599343 e-013$ & $4.44089(-16)$ & $1.879040 e-010$ \\
0.7 & 0.6476559551406433 & $3.994582 e-013$ & $8.8817(-16)$ & $1.776835 e-010$ \\
0.8 & 0.6648399769821803 & $4.342082 e-013$ & $4.44089(-16)$ & $1.724676 e-010$ \\
0.9 & 0.6811859241891134 & $4.647394 e-013$ & $4.44089(-16)$ & $1.847545 e-010$ \\
1.0 & 0.6967346701436833 & $4.911627 e-013$ & $7.77156(-16)$ & $3.005770 e-010$ \\
\hline
\end{tabular}

\subsection{Discussion of Results}

We have considered four numerical examples in this paper. Problem 1 was solved by [9], where they proposed a hybrid block method of order seven and adopted classical Range Kutta to provide the starting values. We solved this problem as shown in Table 1. Problem II was solved by [10]. Problem III and IV were soled by [11] where they proposed a block method of order six, combining power series and exponential function as their approximate solution. The results are shown in Tables III and IV. It has been shown clearly that our method gave better approximation than the existing methods.

\section{Conclusion}

We have proposed a new method that harnesses the properties of the Predictor Corrector method and the Block method. The results affirm the claims of [7] and [8] as discussed in section one. We have equally established that increasing the interpolation points with the same block predictor improves the method. It has been established in literature that the higher the order of a numerical scheme, the higher the accuracy. In our future correspondence, we shall consider a case when the corrector gives results at a non-overlapping interval.

\section{Competing Interests}

The authors declare that no competing interests exist.

\section{References}

1. Awoyemi D.O., A Class of Continuous Method for General Second Order Initial Value Problems in Ordinary Differential Equation. J. of Computer Math, 1999; (72):29-37

2. Onumanyi P, Awoyemi D.O, Jator S.N, and Serisena U.W, New Linear Multistep Method With Constant Coefficient for First Order Initial Value Problems J.N.M.S., 1994;13(7): 37 -51

3. Adesanya A.O, Anake T.A, Oghoyon G.J, Continuous Implicit Method for the Solution of General Second Order Ordinary Differential Equation. J. Nigerian Association of Mathematical Physics, 2009; (40):71-78.

4. Kayode S.J and Awoyemi D.O, A Multi derivative Collocation Method for fifth Order Ordinary Differential Equation. J. of Mathematics and Statistics. 2010; 6(1): 60-63.

5. Milne W.E, Numerical Solution of Differential equation, Wiley Publisher, New York;1953 
6. Adesanya, O A., Odekunle, M.R. and Anake, T. A. 3 Step Continuous Block Predictor Corrector Method for the Solution of General Second Order Ordinary Differential Equations. Journal of Nigerian Association of Mathematical Physics, 21, 2012: 477 - 482

7. Anake T. A, Awoyemi, D. O and Adesanya A. O. One-Step Implicit Hybrid Block Method for the Direct Solution of General Second Order Ordinary Differential Equations. IAENG International Journal of Applied Mathematics, 2012: 42(4):224 -228

8. Adesanya A Olaide, Odekunle M. Remilekun and James A. Adewale, Order seven continuous hybrid method for the solution of first order ordinary differential equations. Canadian Journal on Science and Engineering Mathematics Vol. 3 No. 4: 2012;

9. Areo E.A, Ademiluyi R.A, and Babatola P.O, Three-step hybrid linear multistep method for the solution of first order initial value problem in ordinary differential equation ,J.N.A.M.P, 2011; (19):155-158.

10. Yahaya Y.A. and Umar Mohammed. A family of implicit 4-step block Hybrid Collocation method for accurate and efficient solution of ODEs, Nigerian Journal of Mathematics and Applications. 2010; Vol.20: pp43-52.

11. Sunday J. Odekunle M.R. and Adeyanya A.O. Order six block integrator for the solution of first-order ordinary differential equations, IJMSC , 2013;Vol.3 (1).1: 87-96.

(C)2014 James \& Adesanya; This is an Open Access article distributed under the terms of the Creative Commons Attribution License http://creativecommons.org/licenses/by/3.0, which permits unrestricted use, distribution, and reproduction in any medium, provided the original work is properly cited.

\section{Peer-review history:}

The peer review history for this paper can be accessed here (Please copy paste the total link in your browser address bar)

www.sciencedomain.org/review-history.php?iid=370\&id=6\&aid=3374 